\title{
IS THE COSMOLOGICAL CONSTANT NON-ZERO?
}

\author{
Paul H. Frampton \\ Department of Physics and Astronomy, \\ University of North Carolina, \\ Chapel Hill, NC 27599-3255.
}

\section{THE ISSUE OF $\Lambda$}

The present talk is similar to one at COSMO-98 last month. As is well explained in standard reviews of the cosmological constant $[1,2]$ the theoretical expectation for $\Lambda$ exceeds its observational value by 120 orders of magnitude.

In 1917, Einstein looked for a static solution of general relativity for cosmology and added a new $\lambda$ term:

$$
R_{\mu \nu}-\frac{1}{2} g_{\mu \nu} R-\lambda g_{\mu \nu}=-8 \pi G T_{\mu \nu}
$$

A $\lambda>0$ solution exists with $\rho=\frac{\lambda}{8 \pi G}$, radius $r\left(S_{3}\right)=(8 \pi \rho G)^{-1 / 2}$ and mass $M=$ $2 \pi^{2} r^{3} \rho=\frac{\pi}{4 \sqrt{\lambda} G}$.

In the 1920's the universe's expansion became known (more red shifts than blue shifts). In 1929, Hubble enunciated his law that recession velocity is proportional to distance.

Meanwhile Friedmann (1922) discovered the now-standard non-static model with metric:

$$
d s^{2}=d t^{2}-R^{2}(t)\left[\frac{d r^{2}}{1-k r^{2}}+r^{2}\left(d \theta^{2}+\sin ^{2} \theta d \phi^{2}\right)\right]
$$

In 1923, Einstein realized the dilemna. He wrote to his friend Weyl: "If there is no quasi-static world, then away with the cosmological term".

Setting $\Lambda=0$ does not increase symmetry. In fact, the issue is one of vacuum energy density as follows:

In vacuum:

$$
<T_{\mu \nu}=-<\rho>g_{\mu \nu}
$$

which changes the $\lambda_{\text {eff }}$ by:

$$
\lambda_{e f f}=\lambda+8 \pi G<\rho>
$$

or equivalently:

$$
\rho_{V}=<\rho>+\frac{\lambda}{8 \pi G}=\frac{\lambda_{e f f}}{8 \pi G}
$$


The observational upper limit on $\lambda$ comes from:

$$
\left(\frac{d R}{d t}\right)^{2}=-k+\frac{1}{3} R^{2}(8 \pi G \rho+\lambda)
$$

which expresses conservation of energy and leads to the upper bound $\left|\lambda_{\text {eff }}\right| \leq H_{0}^{2}$.

This translates into $\left|\rho_{V}\right| \leq 10^{-29} \mathrm{~g} / \mathrm{cm}^{3}$. In high-energy units we use $1 \mathrm{~g} \sim 10^{33} \mathrm{eV}$ and $(1 \mathrm{~cm})^{-1} \sim 10^{-4} \mathrm{eV}$ to rewrite $\left|\rho_{V}\right| \leq[(1 / 100) \mathrm{eV}]^{4}$

A "natural" value in quantum gravity is:

$$
\left|\rho_{V}\right|=\left(M_{\text {Planck }}\right)^{4}=\left(10^{28} e V\right)^{4}
$$

which is $10^{120}$ times too big. This has been called the biggest error ever made in theoretical physics!

Even absent the $\left(M_{\text {Planck }}\right)^{4}$ term field theory with spontaneous symmetry breaking leads one to expect $\langle\rho\rangle \gg[(1 / 100) e V]^{4}$. As examples, QCD confinement suggests $<\rho>\sim(200 \mathrm{MeV})^{4}$, which is $10^{40}$ times too big and electroweak spontaneous symmetry breaking would lead to $\langle\rho\rangle \sim(250 \mathrm{GeV})^{4}$ which is $10^{52}$ times too big. This is the theoretical issue. I will briefly mention four approaches to its solution.

\section{(1) Supersymmetry, Supergravity, Superstrings.}

According to global supersymmetry:

$$
\left\{Q_{\alpha}, Q_{\beta}^{\dagger}\right\}_{+}=\left(\sigma_{\mu}\right)_{\alpha \beta} P^{\mu}
$$

and with unbroken supersymmetry:

$$
Q_{\alpha}\left|0>=Q_{\beta}^{\dagger}\right| 0>=0
$$

which implies a vanishing vacuum value for $\left\langle P_{\mu}\right\rangle$ and hence zero vacuum energy as required for vanishing $\Lambda$.

With global supersymmetry promoted to local supersymmetry the expression for the potential is more complicated than this (one can even have $V<0$ ).

When supersymmetry is broken, however, at $\geq 1 \mathrm{TeV}$ one expects again that $\left|\rho_{V}\right|>(1 T e V)^{4}$ which is $10^{54}$ times too big.

So although unbroken supersymmetry looks highly suggestive, broken supersymmetry does not help. The same is generally true for superstrings.

One new and exciting approach - still in its infancy - involves the compactification of the Type IIB superstring on a manifold $S^{5} \times A d S_{5}$ and give rise to a 4-dimensional $\mathcal{N}=4 \mathrm{SU}(\mathrm{N})$ supersymmetric Yang-Mills theory, known to be conformal. Replacing $S^{5}$ by an orbifold $S^{5} / \Gamma$ can lead to $\mathcal{N}=0$ non-supersymmetric $\mathrm{SU}(\mathrm{N})$ gauge theory and probably (this is presently being checked; see e.g. [3]) retain conformal symmetry. If so one may achieve $\langle\rho\rangle=0$ without supersymmetry.

\section{(2) Quantum Cosmology.}

The use of wormholes to derive $\Lambda \rightarrow 0$ has been discredited because of (a) the questionable use of Euclidean gravity, (b) wormholes, if they exist, become macroscopically large and closely-packed, at variance with observation. 


\section{(3) Changed Gravity.}

An example of changing gravity theory [4] is to make $g=\operatorname{det}_{\mu \nu}$ non-dynamical in the generalized action:

$$
S=-\frac{1}{16 \pi G} \int d x[R+L(g-1)]
$$

where $\mathrm{L}$ is a Lagrange multiplier. One then finds by variation that $R=-4 \Lambda=$ constant. Minimizing the action gives $\Lambda=2 \sqrt{6} \pi / \sqrt{V}$ where $V$ is the spacetime volume.

In the path integral

$$
Z=\int d \mu(\Lambda) \exp (3 \pi / G \Lambda)
$$

the value $\Lambda \rightarrow 0^{+}$is exponentially favored.

\section{(4) The Anthropic Principle.}

If $\Omega_{\Lambda} \gg 1$ rapid exponential expansion prohibits gravitational condensation to clumps of matter. This requires $\Omega_{\Lambda}<400$.

On the other hand if $\Omega_{\Lambda} \ll 0$ the universe collapses at a finite time, and there is not enough time for life to evolve. For example, if $\Lambda=-\left(M_{\text {Planck }}\right)^{4}$, $\mathrm{R}$ reaches only $0.1 \mathrm{~mm}\left(10^{-30}\right.$ of its present value). Taken together these two considerations lead to

$$
-1 \leq \Omega_{\Lambda} \leq 400
$$

- quite a strong constraint.

This shows how important it is to life that $\Lambda$ is very much closer to zero than to $\left(M_{\text {Planck }}\right)^{4}$ or even $E^{4}$ where $E$ is any vacuum energy scale familiar to High Energy physics.

\section{CBR TEMPERATURE ANISOTROPY}

The Cosmic Background Radiation (CBR) was discovered [5] in 1965 by Penzias and Wilson. But detection of its temperature anisotropy waited until 1992 when $[6,7]$ the Cosmic Background Explorer (COBE) satellite provided impressive experimental support for the Big Bang model. COBE results are consistent with a scale-invariant spectrum of primordial scalar density fluctuations, such as might be generated by quantum fluctuations during inflation $[8,9,10,11,12,13,14]$. COBE's success inspired many further experiments with higher angular sensitivity than COBE $\left(\sim 1^{\circ}\right)$.

NASA has approved a satellite mission MAP (Microwave Anisotropy Probe) for 2000. ESA has approved the Planck surveyor - even more accurate than MAP - a few years later in 2005 .

With these experiments, the location of the first accoustic (Doppler) peak and possible subsequent peaks will be resolved.

The Hot Big Bang model is supported by at least three major triumphs:

- the expansion of the universe

- the cosmic background radiation

- nucleosynthesis calculations

It leaves unanswered two major questions: 
- the horizon problem

- the flatness problem

The horizon problem. When the CBR last scattered, the age of the universe was $\sim 100,000 y$. The horizon size at that recombination time subtends now an angle $\sim \pi / 200$ radians. On the celestial sphere there are 40,000 regions never causallyconnected in the unadorned Big Bang model. Yet their CBR temperature is the same to one part in $10^{5}$ - how is this uniformity arranged?

The flatness problem. From the equation (for $\Lambda=0$ )

$$
\frac{k}{R^{2}}=(\Omega-1) \frac{\dot{R}^{2}}{R^{2}}
$$

and evaluate for time $t$ and the present $t-t_{0}$, using $R \sim \sqrt{t} \sim T^{-1}$ :

$$
\left(\Omega_{t}-1\right)=4 H_{0}^{2} t^{2} \frac{T^{2}}{T_{0}^{2}}\left(\Omega_{0}-1\right)
$$

Now for high densities:

$$
\frac{\dot{R}^{2}}{R^{2}}=\frac{8 \pi G \rho}{3} \simeq \frac{8 \pi G g a T^{4}}{6}
$$

where $a$ is the radiation constant $=7.56 \times 10^{-9} \mathrm{erg} \quad \mathrm{m}^{-3} \quad \mathrm{~K}^{-4}$.

From this we find

$$
t(\text { seconds })=\left(2.42 \times 10^{-6}\right) g^{-1 / 2} T(G e V)^{-2}
$$

and thence by substitution in Eq. (14)

$$
\left(\Omega_{t}-1\right)=\left(3.64 \times 10^{-21}\right) h_{0}^{2} g^{-1} T(G e V)^{-2}\left(\Omega_{0}-1\right)
$$

This means that if we take, for example, $t=1$ second when $T \simeq 1 \mathrm{MeV}$, then $\left|\Omega_{t}-1\right|$ must be $<10^{-14}$ for $\Omega_{0}$ to be of order unity as it is now. If we go to earlier cosmic time, the fine tuning of $\Omega_{t}$ becomes even stronger if we want the present universe to be compatible with observation. Why then is $\Omega_{t}$ so extremely close to $\Omega_{t}=1$ in the early universe?

Inflation Both the horizon and flatness problems can be solved in the inflationary scenario which has the further prediction (in general) of flatness. That is, if $\Lambda=0$ :

$$
\Omega_{m}=1
$$

or, in the case of $\Lambda \neq 0$ (which is allowed by inflation):

$$
\Omega_{m}+\Omega_{\Lambda}=1
$$

We shall see to what extent this prediction, Eq.(19), is consistent with the present observations.

The goal of the CBR experiments $[15,16,17,18]$ is to measure the temperature autocorrelation function. The fractional perturbation as a function of direction $\underline{\hat{n}}$ is expanded in spherical harmonics:

$$
\frac{\Delta T(\underline{\hat{\mathbf{n}}})}{T}=\sum_{l m} a_{l m} Y_{l m}(\underline{\hat{\mathbf{n}}})
$$


The statistical isotropy and homogeneity ensure that

$$
<a_{l m}^{\dagger} a_{l^{\prime} m^{\prime}}>=C_{l} \delta_{l l^{\prime}} \delta_{m m^{\prime}}
$$

A plot of $C_{l}$ versus $l$ will reflect oscillations in the baryon-photon fluid at the surface of last scatter. The first Doppler, or accoustic, peak should be at $l_{1}=\pi / \Delta \Theta$ where $\Delta \Theta$ is the angle now subtended by the horizon at the time of last scatter: the recombination time at a red-shift of $Z \simeq 1,100$.

The special case $\Lambda=0$

When $\Lambda=0$, the Einstein-Friedmann cosmological equation can be solved analytically (not generally true if $\Lambda \neq 0$ ). We will find $l_{1} \sim 1 / \sqrt{\Omega_{m}}$ as follows. Take:

$$
d s^{2}=d t^{2}-R^{2}\left[\begin{array}{llllll}
d \Psi^{2}+\sinh ^{2} \quad \Psi & d \Theta^{2}+\sinh ^{2} & \Psi & \sin ^{2} & \Theta & d \Phi^{2}
\end{array}\right]
$$

For a geodesic $d s^{2}=0$ and so:

$$
\frac{d \Psi}{d R}=\frac{1}{R}
$$

The Einstein equation is

$$
\left(\frac{\dot{R}}{R}\right)^{2}=\frac{8 \pi G \rho}{3}+\frac{1}{R^{2}}
$$

so that

$$
\dot{R}^{2} R^{2}=R^{2}+a R
$$

with $a=\Omega_{0} H_{0}^{2} R_{0}^{3}$ and hence

$$
\frac{d \Psi}{d R}=\frac{1}{\sqrt{R^{2}+a R}}
$$

This can be integrated to find

$$
\Psi_{t}=\int_{R_{t}}^{R_{0}} \frac{d R}{\sqrt{(R+a / 2)^{2}-(a / 2)^{2}}}
$$

The substitution $R=\frac{1}{2} a(\cosh V-1)$ leads to

$$
\Psi_{t}=\cosh ^{-1}\left(\frac{2 R_{0}}{a}-1\right)-\cosh ^{-1}\left(\frac{2 R_{t}}{a}-1\right)
$$

Using $\sinh \left(\cosh ^{-1} x\right)=\sqrt{x^{2}-1}$ gives

$$
\sinh \Psi_{t}=\sqrt{\left(\frac{2\left(1-\Omega_{0}\right)}{\Omega_{0}}+1\right)^{2}-1}-\sqrt{\left(\frac{2\left(1-\Omega_{0}\right) R_{t}}{\Omega_{0} R_{0}}+1\right)^{2}-1}
$$

The second term of Eq.(29) is negligible as $R_{t} / R_{0} \rightarrow 0$ With the metric of Eq.(22) the angle subtended now by the horizon then is

$$
\Delta \Theta=\frac{1}{H_{t} R_{t} \sinh \Psi_{t}}
$$

For $Z_{t}=1,100$, the red-shift of recombination one thus finds

$$
l_{1}(\Lambda=0) \simeq \frac{2 \pi Z_{t}^{1} / 2}{\sqrt{\Omega_{m}}} \simeq \frac{208.4}{\sqrt{\Omega_{m}}}
$$

This is plotted in Fig. 1 of [19]. 
The general case $\Lambda \neq 0$

When $\Lambda \neq 0$

$$
\dot{R}^{2} R^{2}=-k R^{2}+a R+\Lambda R^{4} / 3
$$

It is useful to define the contributions to the energy density $\Omega_{m}=8 \pi G \rho / 3 H_{0}^{2}, \Omega_{\Lambda}=$ $\Lambda / 3 H_{0}^{2}$, and $\Lambda_{C}=-k / H_{0}^{2} R_{0}^{2}$. These satisfy

$$
\Omega_{m}+\Omega_{\Lambda}+\Omega_{C}=1
$$

Then

$$
l_{1}=\pi H_{t} R_{t} \sinh \Psi_{t}
$$

where

$$
\Psi_{t}=\sqrt{\Omega_{C}} \int_{1}^{\infty} \frac{d w}{\sqrt{\Omega_{\Lambda}+\Omega_{C} w^{2}+\Omega_{m} w^{3}}}
$$

After changes of variable one arrives at

$$
l_{1}=\pi \sqrt{\frac{\Omega_{0}}{\Omega_{C}}} \sqrt{\frac{R_{0}}{R_{t}}} \sinh \left(\sqrt{\Omega_{C}} \int_{1}^{\infty} \frac{d w}{\sqrt{\Omega_{\Lambda}+\Omega_{C} w^{2}+\Omega_{m} w^{3}}}\right)
$$

(For positive curvature $(k=+1)$ replace $\sinh$ by $\sin$ ). For the case $k=0$, the flat universe predicted by inflation, with $\Omega_{C}=0$ Eq.(36) reduces to

$$
l_{1}=\pi \sqrt{\Omega_{m}} \sqrt{\frac{R_{0}}{R_{t}}} \int_{1}^{\infty} \frac{d w}{\sqrt{\Omega_{\Lambda}++\Omega_{m} w^{3}}}
$$

These are elliptic integrals, easily do-able by Mathematica. They resemble the formula for the age of the universe:

$$
A=\frac{1}{H_{0}} \int_{1}^{\infty} \frac{d w}{w \sqrt{\Omega_{\Lambda}+\Omega_{C} w^{2}+\Omega_{m} w^{3}}}
$$

In Fig. 2 of [19] there is a plot of $l_{1}$ versus $\Omega_{m}$ for $\Omega_{C}=0$. In Fig. 3 are the main result of the iso- $l_{1}$ lines on a $\Omega_{m}-\Omega_{\Lambda}$ plot for general $\Omega_{C}$ with values of $l_{1}$ between 150 and 270 in increments $\Delta l_{1}=10$. The final Fig. 4 of [19] gives a three-dimensional plot of $\Omega_{m}-\Omega_{\Lambda}-l_{1}$.

We can look at the cumulative world data on $C_{l}$ versus $l$. Actually even the existence of the first Doppler peak is not certain but one can see evidence for the rise and the fall of $C_{l}$. In Fig. 2 of [20] we see such 1998 data and with some licence say that $150 \leq l_{1} \leq 270$.

The exciting point is that the data are expected to improve markedly in the next decade. In Fig. 3 of [20] there is an artist's impression of both MAP data (expected 2000) and Planck data(2006); the former should pin down $l_{1}$ with a small error and the latter is expected to give accurate values of $C_{l}$ out to $l=1000$.

But even the spectacular accuracy of MAP and Planck will specify only one iso- $l_{1}$ line in the $\Omega_{m}-\Omega_{\Lambda}$ plot and not allow unambiguous determination of $\Omega_{\Lambda}$.

Fortunately this ambiguity can be removed by a completely independent set of observations. 


\section{HIGH-Z SUPERNOVAE IA.}

In recent years several supernovae (type IA) have been discovered with high redshifts $Z>0.3$ (at least 50 of them). An example of a high red-shift is $Z=0.83$. How far away is that in cosmic time? For matter-domination

$$
\left(\frac{R_{0}}{R_{t}}\right)=\left(\frac{t_{0}}{t}\right)^{2 / 3}=(Z+1)
$$

so the answer is $t=t_{0} / 2.83$. For $t_{0}=14 G y$ this implies $t \simeq 6 G y$. Thus this supervova is older than our Solar System and the distance is over half way back to the Big Bang!

These supernovae were discovered $[21,22]$ by a $4 \mathrm{~m}$ telescope then their light-curve monitored by the $10 \mathrm{~m}$ telescope KEK-II on Mauna Kea, Hawaii and/or the Hubble Space Telescope. The light curve is key, because study of nearby supernovae suggests that the breadth of the light curve i.e. the fall in luminosity in 15 days following its peak is an excellent indicator of absolute luminosity. Broader (slower) light curves imply brighter luminosity. Clever techniques compare the SN light-curve to a standard template.

It is worth pointing out that although these SN are very far away - over $50 \%$ back to the Big Bang they do not penetrate as far back as the CBR discussed earlier which goes $99.998 \%$ back to the Big Bang (300,000y out of 14,000,000,000y).

Because of the high $Z$, just one of these observations, and certainly 50 or more of them, have great influence on the estimation of the deceleration parameter $q_{0}$ defined by

$$
q_{0}=-\frac{\ddot{\mathrm{R}} R}{\dot{R}^{2}}
$$

which characterizes departure from the linear Hubble relation $Z=\frac{1}{H_{0}} d$. In the simplest cosmology $(\Lambda=0)$ one expects that $q_{0}=+1 / 2$, corresponding to a deceleration in the expansion rate.

The startling result of the high-Z supernovae observations is that the deceleration parameter comes out negative $q_{o} \simeq-1 / 2$ implying an accelerating expansion rate.

Now if the only sources of vacuum energy driving the expansion are $\Omega_{m}$ and $\Omega_{\Lambda}$ there is the relationship

$$
q_{0}=\frac{1}{2} \Omega_{m}-\Omega_{\Lambda}
$$

So we add a line on the $\Omega_{m}-\Omega_{\Lambda}$ plot corresponding to Eq.41) with $q_{0}=-1 / 2$. Such a line is orthogonal to the iso- $l_{1}$ lines from the CBR Doppler peak and the intersection gives the result that values $\Omega_{m} \simeq 0.3$ and $\Omega_{\Lambda} \simeq 0.7$ are favored. It is amusing that these values are consistent with Eq.(19) but the data strongly disfavor the values $\Omega_{m}=1$ of Eq.(18).

Note that a positive $\Omega_{\Lambda}$ acts like a negative pressure which accelerates expansion - a normal positive pressure implies that one does work or adds energy to decrease the volume and increase the pressure: a positive cosmological constant implies, on the other hand, that increase of volume goes with increase of energy, only possible if the pressure is negative. 


\section{QUINTESSENCE.}

The non-zero value $\Omega_{\Lambda} \simeq 0.7$ has two major problems:

- Its value $(1 / 100 \mathrm{eV})^{4}$ is unnaturally small.

- At present $\Omega_{m}$ and $\Omega_{\Lambda}$ are the same order of magnitude implying that we live in a special era.

Both are addressed by quintessence, an inflaton field $\Phi$ taylored so that $T_{\mu \nu}(\Phi)=$ $\Lambda(t) g_{\mu \nu}$. The potential $V(\Phi)$ may be

$$
V(\Phi)=M^{4+\alpha} \Phi^{-\alpha}
$$

or

$$
V(\Phi)=M^{4}(\exp (M / \Phi)-1)
$$

where $M$ is a parameter [23].

By arranging that $\rho_{\Phi}$ is a little below $\rho_{\gamma}$ at the end of inflation, it can track $\rho_{\gamma}$ and then (after matter domination) $\rho_{m}$ such that $\Omega_{\Lambda\left(t_{0}\right)} \sim \Omega_{m}$ is claimed [24] not to require fine-tuning. The subject is controversial because, by contrast to [24], [25] claim that slow-roll inflation and quintessence require fine-tuning at the level of $1 \mathrm{in} 10^{50}$.

More generally, it is well worth examining equations of state that differ from the one $(\omega=p / \rho=-1)$ implied by constant $\Lambda$. Quintessence covers the possibilities $-1<\omega \leq 0$.

\section{SUMMARY.}

Clearly more data are needed for both the CBR Doppler peak and the high-Z supernovae. Fortunately both are expected in the forseeable future.

The current analyses favor $\Omega_{\Lambda} \simeq 0.7$ and $\Omega_{m} \simeq 0.3$.

Of course, $\Lambda$ is still 120 orders of magnitude below its natural value, and 52 orders of magnitude below $(250 \mathrm{GeV})^{4}$ and that theoretical issue remains.

The non-zero $\Lambda$ implies that we live in a special cosmic era: $\Lambda$ was negligible in the past but will dominate the future giving exponential growth $R \sim e^{\Lambda t}, t \rightarrow \infty$. This cosmic coincidence is addressed by quintessence.

The principal point of our own work in [19] is that the value of $l_{1}$ depends almost completely only on the geometry of geodesics since recombination, and little on the details of the accoustic waves, since our iso- $l_{1}$ plot agrees well with the numerical results of White et al. [26].

\section{ACKNOWLEDGEMENT}

This work was supported in part by the US Department of Energy under Grant No. DE-FG05-85ER41036.

\section{REFERENCES}

1. S. Weinberg, Rev. Mod. Phys. 61, 1 (1989).

2. Y.J. Ng, Int. J. Mod. Phys. D1, 145 (1992).

3. P.H. Frampton, hep-th/9812117. 
4. P.H. Frampton, Y. J. Ng and H. Van Dam, J. Math. Phys. 33, 3881 (1992).

5. A.A. Penzias and R.W. Wilson, Ap. J. 142, 419 (1965).

6. G.F. Smoot et al., Ap. J. Lett. 396, L1 (1992).

7. K. Sanga et al., Ap. J. 410, L57 (1993).

8. J.M. Bardeen, P.J. Steinhardt and M.S. Turner, Phys. Rev. D28, 679 (1983).

9. A.A. Storobinsky, Phys. Lett. B117, 175 (1982).

10. A.H. Guth and S.-Y. Pi, Phys. Rev. Lett. 49, 1110 (1982).

11. S.W. Hawking, Phys. Lett. B115, 295 (1982)

12. A.H. Guth, Phys. Rev. D28, 347 (1981).

13. A.D. Linde, Phys. Lett. B108, 389 (1982).

14. A. Albrecht and P.J. Steinhardt, Phys. Rev. Lett. 48, 1220 (1982).

15. R.L. Davis, H.M. Hodges, G.F. Smoot, P.J. Steinhardt and M.S. Turner, Phys. Rev. Lett. 69, 1856 (1992).

16. J.R. Bond,R. Crittenden, R.L.Davis, G. Efstathiou and P.J. Steinhardt, Phys. Rev. Lett. 72, 13 (1994).

17. P.J. Steinhardt, Int. J. Mod. Phys. A10, 1091 (1995).

18. M. Kamionkowski and A. Loeb, Phys. Rev. D56, 4511 (1997).

19. P.H. Frampton, Y.J. Ng and R. Rohm. Mod. Phys. Lett. A13, 2541 (1998).

20. M. Kamionkowski. astro-ph/980316.

21. S.J. Perlmutter et al., (The Supernova Cosmology Project). astro-ph/9608199.

22. S.J. Perlmutter et al., (The Supernova Cosmology Project). astro-ph/9712219.

23. P.J.E. Peebles and B. Ratra, Ap. J. Lett. 325, L17 (1988).

24. I. Zlatev, L. Wang and P.J. Steinhardt. astro-ph/9807008.

25. C. Kolda and D. Lyth. hep-ph/9811375.

26. M. White and D. Scott, Ap.J. 459, 415 (1996), astro-ph/9508157. W. Hu and M. White, Ap. J. 471, 30 (1996), astro-ph/9602019; Phys. Rev. Lett. 77, 1687 (1996), astro-ph/9602020. M.White, Ap. J. 506, 495 (1998), astro-ph/9802295. 